\title{
REMARKS ON THE TOPOLOGY OF FOLDS
}

\author{
SHIGEKI KIKUCHI AND OSAMU SAEKI
}

(Communicated by Thomas Goodwillie)

\begin{abstract}
We give some necessary conditions for a closed manifold to admit a smooth map into an Euclidean space with only fold singular points.
\end{abstract}

\section{INTRODUCTION}

Let $f: M^{n} \rightarrow \mathbf{R}^{p} \quad(n \geq p)$ be a smooth map of a closed $n$-dimensional manifold into the $p$-dimensional Euclidean space. We say that a singular point $q \in M$ of $f$ is a fold singular point of index $\lambda$ if $f$ has the normal form as follows for some local coordinate systems around $q$ and $f(q)$ :

$$
\begin{aligned}
& y_{i} \circ f=x_{i} \quad(1 \leq i \leq p-1), \\
& y_{p} \circ f=-x_{p}^{2}-\cdots-x_{p+\lambda-1}^{2}+x_{p+\lambda}^{2}+\cdots+x_{n}^{2},
\end{aligned}
$$

where $0 \leq \lambda \leq n-p+1$ is an integer. If $\lambda=0$ or $n-p+1$, we say that $q$ is a definite fold singular point. Our main results of this paper are as follows.

Theorem 1.1. If a closed $n$-dimensional manifold $M^{n}$ admits a smooth map $f: M^{n} \rightarrow \mathbf{R}^{p} \quad(n \geq p)$ with only definite fold singular points, then it is smoothly null-cobordant. If, in addition, $M^{n}$ is oriented, it is null-cobordant in the oriented category.

Theorem 1.2. Let $M^{n}$ be a closed $n$-dimensional manifold with odd Euler number. If $M^{n}$ admits a smooth map $f: M^{n} \rightarrow \mathbf{R}^{p} \quad(n \geq p)$ with only fold singular points, then $p=1,3$, or 7 .

Note that Theorem 1.1 has been proved in [7, Corollary 3.3] when $n>p$. Note also that the fact that $p$ must be odd in Theorem 1.2 has been obtained in $[8$, Theorem 1$]$.

\section{PROOFS OF THE THEOREMS}

Proof of Theorem 1.1. If $n>p$, the theorem is proved in [7]. Furthermore, if $n=p$ and $M^{n}$ is oriented, then $M^{n}$ is stably parallelizable by [2] (see also [7, Proposition 7.4]), and hence it is null-cobordant in the oriented category, since

Received by the editors November 24, 1992.

1991 Mathematics Subject Classification. Primary 57R45; Secondary 57R20, 57R42.

The second author is partially supported by the Grants-in-Aid for Encouragement of Young Scientists (no. 04740007), The Ministry of Education, Science, and Culture, Japan. 
all the characteristic classes vanish. Thus we assume that $n=p$ and $M^{n}$ is nonorientable. In this case, by Corollary 7.5 of [7], the Stiefel-Whitney classes $w_{i}\left(M^{n}\right)$ vanish for all $i \geq 2$. Thus it suffices to prove the following.

Lemma 2.1. Let $M^{n}$ be a closed n-dimensional manifold whose Stiefel-Whitney classes $w_{i}\left(M^{n}\right)$ vanish for all $i \geq 2$. Then it is null-cobordant.

Proof. Let $\bar{w}(M)=1+\bar{w}_{1}(M)+\cdots+\bar{w}_{n}(M) \in H^{*}\left(M ; \mathbf{Z}_{2}\right)$ be the dual StiefelWhitney class of $M$. By the hypothesis, we see easily that

$$
\bar{w}(M)=1+w_{1}(M)+w_{1}(M)^{2}+\cdots+w_{1}(M)^{n}
$$

and, in particular, that

$$
\bar{w}_{n}(M)=w_{1}(M)^{n} .
$$

On the other hand, it is well known that $\bar{w}_{n}(M)$ always vanishes for every $n$-dimensional manifold $M$, since, by Whitney [10], it can be immersed into $\mathbf{R}^{2 n-1}$ if $n \geq 2$. (See also [6, p. 136].) Thus we see that $w_{1}(M)^{n}=0$ and, hence, that the corresponding Stiefel-Whitney number vanishes. By our hypothesis, all the other Stiefel-Whitney numbers also vanish. Thus $M$ is nullcobordant. This completes the proof of Lemma 2.1 and, hence, of Theorem 1.1 .

Proof of Theorem 1.2. Let $S(f)$ be the singular set of the smooth map $f: M^{n} \rightarrow$ $\mathbf{R}^{p}$; i.e., $S(f)=\left\{q \in M\right.$; rank $\left.d f_{q}<p\right\}$. It is easy to see, by the definition of a fold singular point, that $S(f)$ is a $(p-1)$-dimensional closed submanifold of $M$ and that $f \mid S(f): S(f) \rightarrow \mathbf{R}^{p}$ is a codimension-1 immersion. On the other hand, by Fukuda [3] and our hypothesis, we see that the Euler number of $S(f)$ is odd. In particular, $S(f)$ is not null-cobordant. Then, by a theorem of Brown [1] (see also [5]), we see that $S(f)$ must be cobordant to $\mathbf{R} P^{0}, \mathbf{R} P^{2}$, or $\mathbf{R} P^{6}$. This shows that $p-1=0,2$, or 6 . This completes the proof of Theorem 1.2 .

Remark 2.2. Note that, for $p=1,3$, and 7, there exist smooth maps $f: M^{n} \rightarrow$ $\mathbf{R}^{p}$ with only fold singular points such that $M^{n}$ has odd Euler number. Such an example can be found in [8, Example 3.7], where a smooth map $M^{4} \rightarrow \mathbf{R}^{3}$ as above is explicitly constructed. Using a similar method and the fact that $\mathbf{R} P^{6}$ can be immersed into $\mathbf{R}^{7}$, for $p=3,7$ and $n>p$ with $n$ even, we can construct a smooth map $f: M^{n} \rightarrow \mathbf{R}^{p}$ with only fold singular points such that $M^{n}$ has odd Euler number. Note that for $p=1$, this is obvious, since smooth maps $f: M^{n} \rightarrow \mathbf{R}$ with only fold singular points are nothing but Morse functions.

Remark 2.3. Sakuma conjectures that if $M^{n}$ is orientable and has odd Euler number, then $M^{n}$ does not admit any smooth map $f: M^{n} \rightarrow \mathbf{R}^{p} \quad(n \geq p \geq 2)$ with only fold singular points (cf. [9]). Theorem 1.2 shows that this conjecture is true for $p \neq 3,7$. Note that the examples constructed in Remark 2.2 are not orientable. Thus, for $p=3,7$, the problem of Sakuma is still open.

Remark 2.4. In Theorem 1.2, we cannot replace the condition that $M^{n}$ has odd Euler number with the condition that $M^{n}$ is not null-cobordant. In fact, there exist manifolds which are not null-cobordant and which admit smooth maps into Euclidean spaces $\mathbf{R}^{p}(p \neq 1,3,7)$ with only fold singular points. For example, the connected sum of two copies of the complex projective plane 
$\mathbf{C} P^{2} \# \mathbf{C} P^{2}$ admits a smooth map into $\mathbf{R}^{2}$ with only fold singular points (see [4]).

We end this paper by a remark concerning the cobordism class of the singular set. In the proof of Theorem 1.2, we used the fact that if $M^{n}$ has odd Euler number, then the singular set $S(f)$ is not null-cobordant. In fact, the converse of this fact also holds as follows.

Proposition 2.5. Let $f: M^{n} \rightarrow \mathbf{R}^{p} \quad(n \geq p)$ be a smooth map of a closed $n$-dimensional manifold $M^{n}$ into the Euclidean space with only fold singular points. Then the following are equivalent.

(1) $M^{n}$ has even Euler number.

(2) $S(f)$ has even Euler number.

(3) $S(f)$ is null-cobordant.

Furthermore, if $S(f)$ is oriented, it is always stably parallelizable. In particular, it is null-cobordant in the oriented category.

Proof. The equivalence of (1) and (2) is easily seen by the fact that the Euler numbers of $M^{n}$ and $S(f)$ have the same parity [3]. Part (3) obviously implies (2). Thus we have only to show that $S(f)$ is null-cobordant if it has even Euler number. Let $w(S(f)) \in H^{*}\left(S(f) ; \mathbf{Z}_{2}\right)$ be the total Stiefel-Whitney class of $S(f)$. Since $S(f)$ admits a codimension-1 immersion into the Euclidean space, we see easily that

$$
w(S(f))=1+w_{1}(S(f))+w_{1}(S(f))^{2}+\cdots+w_{1}(S(f))^{p-1},
$$

where $w_{1}(S(f)) \in H^{1}\left(S(f) ; \mathbf{Z}_{2}\right)$ is the first Stiefel-Whitney class of $S(f)$. Thus the only Stiefel-Whitney number of $S(f)$ is the one corresponding to $w_{1}(S(f))^{p-1}$, which is zero by our hypothesis. Hence, $S(f)$ is null-cobordant.

If $S(f)$ is oriented, then the normal bundle of the immersion $f \mid S(f)$ is trivial and hence $S(f)$ is stably parallelizable. Thus, in this case, $S(f)$ is null-cobordant in the oriented category.

Remark 2.6. In Theorem 1.1, $S(f)$ is orientable. Thus $S(f)$ is always nullcobordant and $M^{n}$ has even Euler number. In fact $S(f)$ bounds a canonical $p$-dimensional orientable manifold, called the Stein factorization of $f$ [7]. Furthermore, in the above proposition, if $p \neq 1,3,7$, then (1)-(3) always hold by Theorem 1.2.

\section{REFERENCES}

1. R. L. W. Brown, A note on immersions up to cobordism, Illinois J. Math. 21 (1977), 240-241.

2. J. M. Èliašberg, On singularities of folding type, Math. USSR-Izv. 4 (1970), 1119-1134.

3. T. Fukuda, Topology of folds, cusps and Morin singularities, A Fete of Topology (Y. Matsumoto, T. Mizutani, and S. Morita, eds.), Academic Press, New York, 1987, pp. 331-353.

4. M. Kobayashi, Simplifying certain mappings from simply connected 4-manifolds into the plane, Tokyo J. Math. 15 (1992), 327-349.

5. A. Liulevicius, Immersions up to cobordism, Illinois J. Math. 19 (1975), 149-164.

6. J. Milnor and J. Stasheff, Characteristic classes, Ann. Math. Stud., no. 76, Princeton Univ. Press, Princeton, NJ, 1974.

7. O. Saeki, Topology of special generic maps of manifolds into Euclidean spaces, Topology Appl. 49 (1993), 265-293.

8. O. Saeki, Notes on the topology of folds, J. Math. Soc. Japan 44 (1992), 551-566. 
9. K. Sakuma, On the topology of simple fold maps, Tokyo J. Math. 17 (1994), 21-31.

10. H. Whitney, The singularities of a smooth n-manifold in (2n-1)-space, Ann. of Math. (2) 45 (1944), 247-293.

Department of Mathematics, Faculty of Science, Hirosaki University, Hirosaki 036, JAPAN

E-mail address: skiku@cc.hirosaki-u.ac.jp

Department of Mathematics, Faculty of Science, Yamagata University, Yamagata 990, JAPAN

Current address: Department of Mathematics, Faculty of Science, Hiroshima University, Higashi-Hiroshima 724, Japan

E-mail address: saeki@math.sci.hiroshima-u.ac.jp 